\title{
Estudo Sobre a Espectroscopia da Hipericina
}

\author{
Lurian B. Barboza, Joaquim D. Motta, Rafael C. Barreto, Harley P. M. Filho, Márcia N. \\ Demarchi \& Lucas Amin
}

A molécula de hipericina é um corante amarelo brilhante extraído das pétalas de Hypericum perforatum. Este corante foi estudado extensivamente em conexão com o seu possível uso como fotossensibilizador em protocolos de terapia fotodinâmica para o tratamento do câncer. Neste trabalho obtivemos geometrias em nível MP2 / aug-cc - PVDZ e em nível AM1 para o corante. Usamos a estrutura AM1 envolvida por várias moléculas de solvente para verificar o efeito do solvente no espectro de absorção. Nessa geometria, os espectros eletrônicos em água e em fase gasosa foram calculados com o método INDO / S. O método INDO / S para a molécula solvatada simula o solvente por uma abordagem combinada de supermoléculas com o modelo contínuo . Fizemos uma simulação da hipericina solvatada em água usando o código GROMACS. A caixa de $3 \mathrm{~nm} 3$ continha uma molécula de hipericina solvatada por 871 moléculas de água. Seguindo o protocolo habitual, procedemos uma equilíbrição inicial da caixa nos conjuntos NpT e NVT. Em seguida, realizamos uma simulação de Dinâmica Molecular de $20 \mathrm{~ns}$ e verificamos uma camada de solvatação de 88-102 moléculas de água através dos gráficos de distribuição radial. Nós extraímos um conjunto de 100 configurações termodinamicamente não correlacionadas da simulação para análise de correlação estatística. Concluímos que a metodologia INDO / S é eficiente para a espectroscopia eletrônica de corantes orgânicos, podendo ser usada em computadores pessoais e com baixo custo computacional. No entanto, uma espectroscopia detalhada é necessária através de simulações de dinâmica molecular em diferentes condições para elucidar o mecanismo biológico deste corante.

Palavras Chave: efeito solvente; hipericina; produto natural; solvatocromismo; terapia fotodinâmica.

The hypericin molecule is a bright yellow dye extracted from the petals of Hypericum perforatum. This dye has been studied extensively in connection to its possible use as photosensitizer in Photodynamic therapy protocols for câncer treatment . In this work we have obtained geometries at MP2/aug-cc-PVDZ and AM1 level for the dye. We use the AM1 strucuture surrounded by a number of solvent molecules for check the solvent effect in absorption spectra. In this geometry, the electronic spectra in water and in gas phase was calculated with the INDO/S method. The INDO/S method for the solvated molecule simulate the solvent by a combined continuum/ supermolecule approach. We have made a simulation of hypericin solvated in water using the GROMACS code. The box of $3 \mathrm{~nm} 3$ contained one hypericin molecule solvated in 871 water molecules. Following the usual protocol, we have proceeded an initial equilibration of the box at both the NpT and NVT ensembles. Next we carried out a MD simulation of $20 \mathrm{~ns}$ and checked a solvation shell of 88-102 water molecules through the radial distribution graphs. We extracted a set of 100 thermodynamically uncorrelated configurations from the simulation for statisctical correlation analysis. We conclude that the INDO / S methodology is very efficient for electronic spectroscopy of organic dyes, can be done on personal computers and with low computational cost. However, a detailed spectroscopy is necessary through simulations molecular dynamics under diferent conditions to elucidate the biological mechanism of this dye .

Keywords: Hypericin; Natural Product; Photodynamic therapy; solvatochromism; solvent effects. 


\section{Introdução}

A molécula de hipericina, um corante amarelo extraído da espécie Hypericum perforatum, vem ganhando espaço nas ultimas décadas em pesquisas relacionadas com Terapia fotodinâmica (PDT) devido a sua notável atividade biológica frente a diversas doenças, desde depressão até redução de tumores. No entanto, os mecanismos de sua atividade biológica continuam não elucidados apesar de vários estudos sobre estes. Dessa forma, a fim de investigar tais mecanismos de forma a elucidar a atividade biológica relatada procurou-se executar cálculos de Mecânica Quântica utilizando as geometrias mais estáveis obtidas para a molécula de hipericina.<smiles></smiles>

Figura 1. Estrutura da Hipericina.

\section{Metodologia}

Inicialmente foram realizados cálculos ab initio em nível MP2/aug-cc-pVDZ para determinar as conformações mais estáveis do corante. Nossos resultados essencialmente reproduzem os resultados de Wieczorek e colaboradores. A seguir, procurou-se caracterizar a estrutura eletrônica do corante, bem como mapear o efeito solvente da água sobre a espectroscopia do corante. Para tanto, utilizamos o método INDO/S para descrição das bandas mais intensas do corante, incluindo o solvente dentro do formalismo de supermoléculas.

No momento estamos explorando a possibilidade de simular o espectro eletrônico com simulações de dinâmica molecular em diferentes solventes utilizando o código GROMACS.

\section{Resultados e Discussão}

Escolhemos como geometria de referência a otimizada em nível AM1.

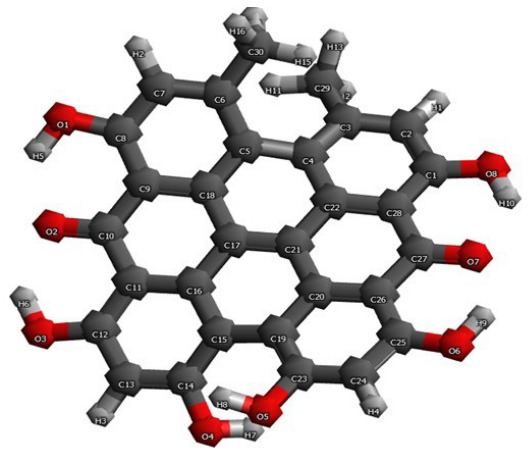

Figura 2. Geometria otimizada por Da Motta.

Usando esta geometria de referência, obtem-se o seguinte esquema para transições em fase gasosa e fase aquosa:

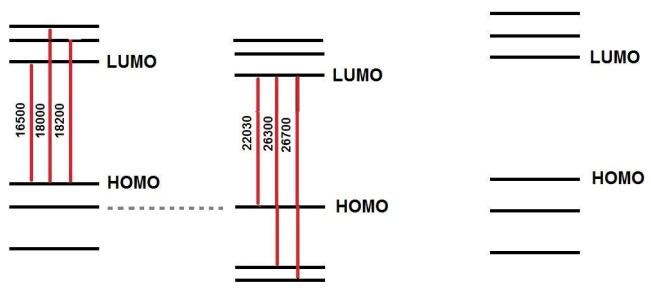

Figura 3. Diagrama representando as transições eletrônicas de mais baixa energia da hipericina calculadas em fase aquosa (incluindo moléculas de solvente em torno do corante, mostrada à esquerda) e em fase gasosa (corante isolado, á direita).

Já conseguimos executar uma dinâmica molecular de 20 ns, numa caixa com 871 moléculas de água, definindo uma camada de solvatação de 88-102 moléculas. A seguir procede-se uma amostragem de 100 configurações não-correlacionadas para análise dos dados estatísticos. Estimou-se uma camada de solvatação, a partir da simulação de $20 \mathrm{~ns}$, em torno de 88-102 moléculas de água a partir de gráficos de distribuição radial. 

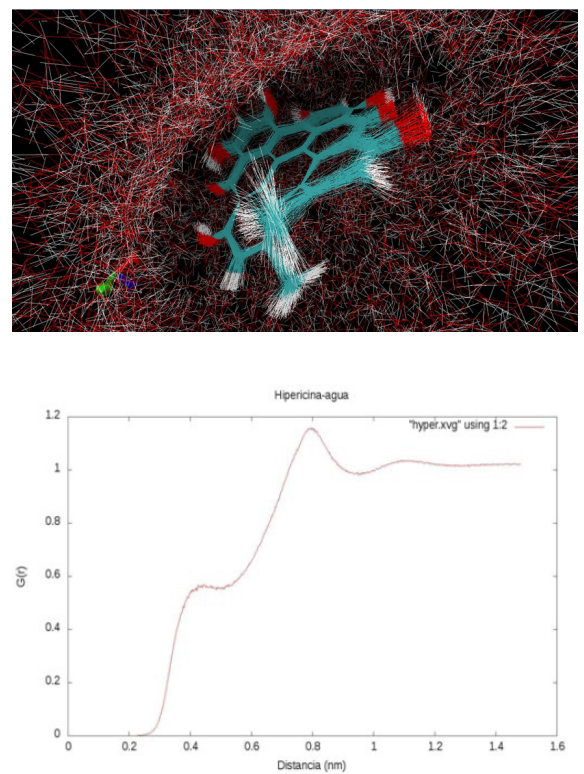

Figura 4. Configurações do corante sobrepostas (á direita) e distribuição radial hipericina-água (á direita).

\section{Conclusões}

A metodologia INDO/S é bastante eficiente para reproduzir espectros eletrônicos de corantes orgânicos, podendo ser realizada em computadores pessoais e com baixo custo computacional. Todavia, um maior detalhamento da espectroscopia através de simulações de dinâmica molecular é necessário para elucidação do mecanismo.

\section{Agradecimentos}

Agradeço ao grupo de pesquisa em Física Computacional do DAFIS-UTFPR pelos recursos que nos proporcionaram os resultados obtidos e aos colegas que contribuíram com seus conselhos.

\section{Referências Bibliográficas}

1. F. Gai, M.J. Fehr, J.W. Petrich, J. Phys. Chem. 98, 8352-8358 (1994).
2. M. Pietrzak, M. Maciejczyk, Z. Wieczorek, Chem. Phys. Lett. 601, 39-44 (2014).

3. R. Guedes, L. Eriksson, J. Photochem. Photobiol. A Chem. 172, $293-$ 299 (2005).

4. A. Darmanyan, W. Jenks, D. Eloy, P. Jardon, J. Phys. Chem. B 103, 3323-31 (1999).

5. GROMACS 4 code, B. Hess, C. Kutzner, D. van der Spoel, E. Lindahl, J. Chem Theory Comput. 4, 435-447 (2008).

6. R.C. Barreto, K. Coutinho, S. Canuto, Phys. Chem. Chem. Phys. 11, 1388-96 (2009).

7. J.E. Ridley and M.C. Zerner, Theor. Chim. Acta (Berlin) 32, 111-134 (1973).

8. C. Robertson, D. Hawkins Evans, J. Photochem. Photobiol. B: Chem. 96, 1-8(2009).

9. R. Yin and M.R. Hamblin, Curr. Med. Chem. 22, 2159-2185 (2015).

10. J.D. Da Motta, R. Bicca de Alencastro, M.C. Zerner, Int. J. Quantum Chem. Quantum Chem. Symp. 28, 361-377 (1994).

\section{Lurian B. Barboza', Joaquim D. Motta ${ }^{1 *}$, Rafael C. Barreto', Harley P. M. Filho', Márcia N. Demarchi ${ }^{1} \&$ Lucas Amin ${ }^{2}$}

\footnotetext{
${ }^{1}$ Departamento de Química, Caixa Postal 19032, Univ. Federal do Paraná (UFPR), Centro Politécnico - Curitiba/PR

2 Departamento de Física, Univ. Tecnológica Federal do Paraná (UTFPR), Av. Sete de Setembro, 3165 - Rebouças Curitiba/PR

*E-mail: quim@ufpr.br
} 